\title{
Whole Animal
}

National Cancer Institute

\section{Source}

National Cancer Institute. Whole Animal. NCI Thesaurus. Code C77665.

Referring to the entire body of an animal. 